\title{
DIAGNÓSTICO E TRATAMENTO DE SÍFILIS DURANTE A GESTAÇÃO: DESAFIO PARA ENFERMEIRAS?
}

\section{Isadora Machado}

Acadêmica de Enfermagem do Centro Universitário de Volta Redonda (UniFOA), Brasil.

\section{Victória Agna Nascimento da Silva}

Acadêmica de Enfermagem do Centro Universitário de Volta Redonda (UniFOA), Brasil.

\section{Renata Martins da Silva Pereira}

Docente do Curso de Enfermagem do Centro Universitário de Volta Redonda (UniFOA). Doutoranda em Enfermagem e Biociências - Universidade Federal do Estado do Rio de Janeiro (UNIRIO), Brasil.

\section{Cristiane Gorgati Guidoreni}

Docente do Curso de Enfermagem do Centro Universitário de Volta Redonda (UniFOA). Doutora em Ciências Universidade Federal de São Paulo (UNIFESP), Brasil.

\section{Mariane de Paula Gomes}

Docente do Curso de Enfermagem do Centro Universitário de Volta Redonda (UniFOA). Mestre em Ensino de Ciências da Saúde e Meio Ambiente (UniFOA), Brasil.
RESUMO: Identificar dificuldades ou facilidades que enfermeiras (os) encontram para realizar o tratamento da síflis na gestante e em seus parceiros sexuais. Pesquisa exploratória, descritiva e qualitativa. Participaram 29 enfermeiras que atuam em Unidades Básicas de Saúde e responderam a um questionário. A maioria (86\%) das enfermeiras realiza o teste rápido, recruta parceiros sexuais das gestantes para realizar o teste e $62 \%$ responderam que administram penicilina benzatina na própria unidade para o tratamento; a oferta do teste rápido na própria unidade e a agilidade de retorno do resultado do exame foram relatadas como principais facilidades encontradas; as dificuldades foram a adesão do parceiro ao tratamento seguido da falta de comprometimento da gestante para seguir o tratamento. $\mathrm{O}$ objetivo foi alcançado e ainda são necessários avanços no tratamento da sífilis a ser realizado pela enfermeira na totalidade dos casos, no que diz respeito à prescrição da penicilina e seu uso nas UBS, e no acolhimento do casal para controle da sífilis na gestação.

PALAVRAS-CHAVE: Síflis; Saúde da mulher; Atenção básica; Pré-natal.

\section{DIAGNOSIS AND TREATMENT OF SYPHILIS DURING PREGNANCY: A CHALLENGE FOR NURSES?}

\begin{abstract}
Identification of difficulties or facilities that nurses have to copy with in the treatment of syphilis in pregnant women and their partners. Current exploratory, descriptive and qualitative research involved 29 nurses from Basic Health Units who answered a questionnaire. Results: most (86\%) nurses applied the fast test and called partners of the pregnant women for tests; $62 \%$ answered they applied benzamine penicillin at the health unit; applying the fast test at the health unit and return agility of exam result were reported as main benefits; difficulties consisted of adhesion of partner and the lack of compromise by the pregnant female to follow the treatment. Aim was reached but several items in the treatment of syphilis should be upgraded, especially the prescription of penicillin and its use in a health unit and the acceptance of the couple for the control of syphilis in pregnancy.
\end{abstract}

KEY WORDS: Syphilis; Women’s health; First care; Pre-natal tests.

\section{INTRODUÇÃO}

Autor correspondente

Renata Martins da Silva Pereira

E-mail: renataenfprofessora@gmail.com

A sífilis continua presente em nosso meio mesmo sendo uma Infecção Sexualmente Transmissível (IST) passível de diagnóstico, 
tratamento e controle. A gestação torna-se um período favorável ao diagnóstico e tratamento adequados, pois a rotina preconizada pelo Ministério da Saúde para o acompanhamento pré-natal conta com vários exames laboratoriais incluindo os testes para síflis. A testagem para síflis deve ocorrer "na primeira consulta do prénatal (idealmente, no primeiro trimestre da gestação), no início do terceiro trimestre ( $28^{\mathrm{a}}$ semana) e no momento do parto ou aborto, independentemente de exames anteriores"1.

Esta infecção é causada pela bactéria Treponema pallidum, cujo sintoma mais comum é uma úlcera indolor na região genital. Na gestação pode ser transmitida pela disseminação hematogênica do treponema pallidium para o concepto por via transplacentária e comprometer a saúde materna e fetal ${ }^{2}$.

A criação e implementação da "Rede Cegonha", pelo Ministério da Saúde, trouxe uma perspectiva de mudança no atendimento à mulher no período gravídico puerperal por parte dos profissionais de saúde, pelo fortalecimento da oferta dos testes rápidos para rastreio de sífilis e HIV (Vírus da Imunodeficiência Humana) nas Unidades de Atenção Básica. A Rede Cegonha visa assegurar à mulher e à criança o direito à atenção humanizada e qualificada durante o pré-natal, parto/ nascimento, período pós-parto e atenção infantil em todos os serviços de saúde do Sistema Único de Saúde (SUS) $)^{2}$.

A Organização Mundial da Saúde (OMS) estima no mundo mais de 1 milhão de casos de Infecções Sexualmente Transmissíveis (IST) por dia. Em especial, a síflis na gestação leva a mais de 300 mil mortes fetais e neonatais por ano no mundo, apresentando um adicional de 215 mil crianças em aumento do risco de morte prematura. A notificação compulsória de gestante com sífilis em todo o território nacional foi instituída por meio da portaria $\mathrm{n}^{\mathrm{o}} 33$ de 14 de julho de 2005 . No Brasil, na última década, observou-se o aumento de notificação de casos de sífilis em gestante que pode ser atribuído, em parte, ao aprimoramento do sistema de vigilância epidemiológica e à ampliação da distribuição de testes rápidos ${ }^{3}$.

No período de 2005 a junho de 2016, foi notificado no Sistema de Informação de Agravos de Notificação
(SINAN) o total de 169.546 casos de síflis em gestantes, dos quais $42,9 \%$ foram casos de mulheres residentes na região Sudeste. Quanto à mortalidade infantil (em menores de 1 ano de idade) por sífilis congênita, no período de 1998 a 2015, o número de óbitos declarados no Sistema de Informação sobre Mortalidade (SIM) foi de 1.903, sendo $826(43,4 \%)$ na região Sudeste (dos quais 568 foram registrados no Estado do Rio de Janeiro, o que corresponde a 29,8\% do Brasil) ${ }^{3}$.

Ainda existem desafios para o controle da doença como pleno acesso aos serviços de saúde, falta de solicitação para realização do exame sorológico das gestantes conforme preconizado, pobreza que impede o acesso pleno ao serviço de saúde, grau de instrução e falta de conhecimento a respeito das infecções sexualmente transmissíveis e seu potencial de complicações e a não abordagem para tratamento e acompanhamento dos parceiros sexuais daquelas mulheres com resultado do teste sorológico positivo ${ }^{4}$.

O tratamento e a adesão dos parceiros sexuais de gestantes com resultado positivo para sífilis são um desafio constante no cotidiano de trabalho dos profissionais que atendem ao pré-natal nas Unidades Básicas de Saúde (UBS). A resistência ao tratamento contribui negativamente para o controle da doença, o que pode ser justificado, principalmente, pela baixa adesão ao serviço de saúde pela população masculina, motivos empregatícios ou falta de conhecimento acerca da importância de cuidar da saúde e das consequências que a doença pode trazer para o indivíduo, o concepto e o casal ${ }^{4}$.

O objetivo da pesquisa foi identificar dificuldades ou facilidades que enfermeiras (os) encontram para realizar o tratamento da sífilis na gestante e em seus parceiros sexuais.

\section{METODOLOGIA}

Trata-se de uma pesquisa descritiva e com abordagem qualitativa, que foi realizada em Unidades Básicas de Saúde da família (UBSF) do Município de Volta Redonda (RJ).

O cenário da pesquisa contou com 18 Unidades de Saúde da Família do município de Volta Redonda (RJ). A Rede de Atenção Básica é constituída por 43 unidades, 
sendo 35 Unidades Básicas de Saúde da Família, oito Unidades Básicas, cinco Clínicas Odontológicas Concentradas e um Núcleo de Apoio à Saúde da Família (NASF), integradas e distribuídas em diferentes bairros, com cobertura de $100 \%$ de atenção básica e $70 \%$ de saúde da família.

Participaram da pesquisa 29 enfermeiras das UBSF. Como critério de inclusão utilizou-se exercício profissional de, no mínimo, um ano na atenção básica e que aceitassem responder ao questionário, após conhecimento dos objetivos da pesquisa.

A coleta de dados foi realizada pela aplicação de questionário, elaborado pelas próprias pesquisadoras, junto às enfermeiras que atuam nas Unidades Básicas de Saúde da Família (UBSF) no período de julho a agosto de 2017. Foram visitadas 18 unidades, selecionadas de forma aleatória. Todas as unidades contam com enfermeiras (os) atendendo o pré-natal de baixo risco.

A análise dos dados foi realizada a partir de estatística descritiva por meio de distribuição de frequências absolutas e relativas, e ainda foi feita correlação entre o conteúdo apresentado nas respostas às perguntas abertas e a literatura atual sobre o tema.

O projeto foi aprovado pelo Comitê de Ética em Pesquisa com Seres Humanos do UniFOA, segundo parecer $n^{\circ}$ 2.168.010, conforme a resolução 466/2012 do Conselho Nacional de Saúde - CNS, que regulamenta os aspectos legais para Pesquisas com Seres Humanos e seguiu a recomendação de assinatura do Termo de Consentimento Livre e Esclarecido (TCLE).

\section{RESULTADOS}

Quanto ao tempo de atuação em UBSF, 16 (55\%) enfermeiras têm mais de cinco anos de atuação, 11 (38\%) tem até cinco anos e duas (7\%) tem um ano de atuação. Destaca-se, ainda, que em relação às especialidades 18 enfermeiras possuem especialização em Saúde da Família, o que representa (62\%) do total de enfermeiras.

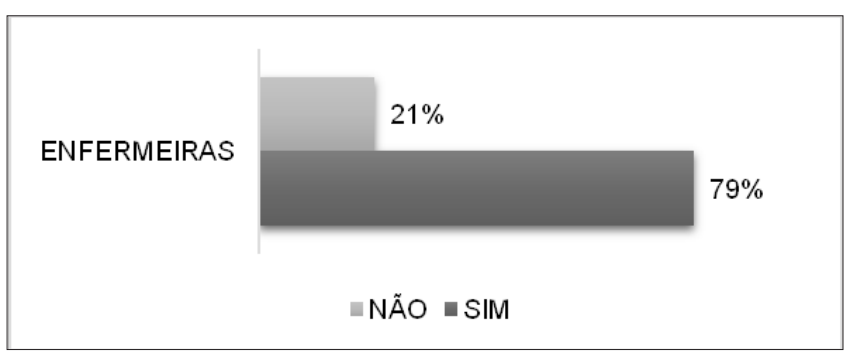

Figura 1. Capacitação para atender ao pré-natal. Fonte: Dados da pesquisa, 2017.

Do total de enfermeiras, 23 (79\%) delas estão habilitadas para atender ao pré-natal de baixo risco e apenas seis (21\%) não possuem nenhum tipo de capacitação nessa área.

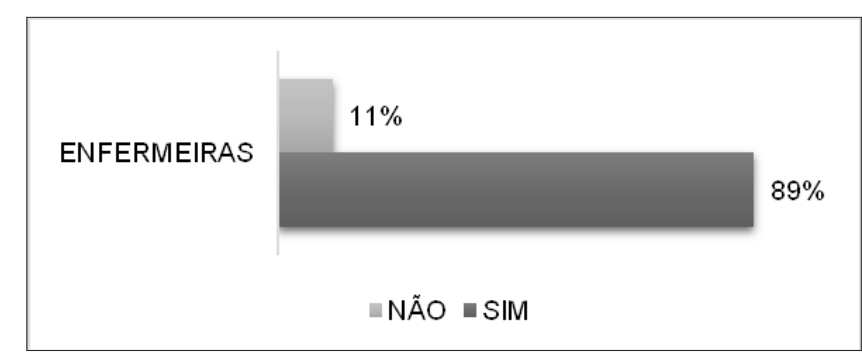

Figura 2. Capacitação específica para diagnóstico e tratamento de sífilis.

Fonte: Dados da pesquisa, 2017.

Na Figura 2 é demonstrado que 26 enfermeiras, das 29 entrevistadas, têm capacitação específica para tratamento e diagnóstico de sífilis, o que representa $89 \%$ do total e apenas $11 \%$ não têm esse tipo de capacitação. O que caracteriza boa adesão das profissionais a cursos e capacitações oferecidas pelo município aos profissionais da atenção básica.

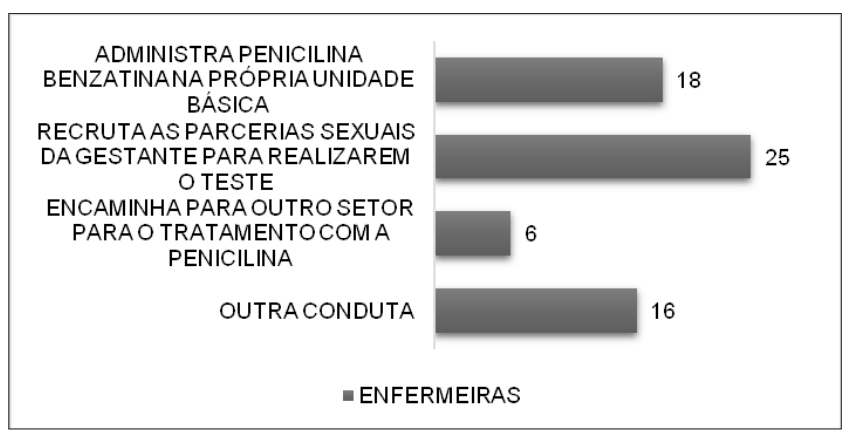

Figura 3. Condutas caso o resultado do teste seja positivo para sífilis

Fonte: Dados da pesquisa, 2017. 
Destaca-se na Figura 3 as condutas adotadas pelas enfermeiras frente a um caso de resultado positivo para sífilis. Observa-se que para a maioria das enfermeiras (25-86\%), há a preocupação em recrutar parceiros sexuais das gestantes para realizarem o teste de sífilis; 18 (62\%) enfermeiras administram penicilina benzatina na própria unidade para o tratamento rápido e eficaz da infecção e ainda seis (21\%) enfermeiras relataram que na falta de medicação na unidade as pacientes são encaminhadas para outro setor, no caso o Centro de Doenças Infecciosas, onde são atendidas por médicos especialistas.

Além das questões fechadas, o questionário também contou com três perguntas abertas relacionadas às facilidades e/ou dificuldades que as enfermeiras encontram no tratamento da sífilis e sobre quais estratégias utilizam para o tratamento e abordagem de parceiros sexuais de gestantes com teste positivo para sífilis.

Sobre a questão da abordagem das gestantes para o rastreamento da síflis na gestação, a maioria delas (55\%) respondeu que faz a abordagem na primeira consulta de pré-natal utilizando a educação em saúde e o acolhimento. Nas unidades que possuíam o teste rápido, as enfermeiras, ofertam o mesmo a todas as gestantes já na primeira consulta de pré-natal; relataram ainda que promovem o encaminhamento das gestantes atendidas para realização dos exames laboratoriais de primeira rotina do pré-natal.

Em relação às facilidades e/ou dificuldades encontradas para o tratamento da sífilis em gestantes, a maior facilidade relatada foi a disponibilidade do teste rápido na própria unidade, sendo uma oportunidade para as enfermeiras detectarem precocemente a sífilis, seguida da disponibilidade da medicação para o tratamento no município. Algumas enfermeiras relataram também que a agilidade por parte do laboratório em liberar os resultados do exame facilitava o diagnóstico. Apenas 10\% das enfermeiras relatam ter fácil aceitação do tratamento por parte da gestante.

Quanto às dificuldades para o tratamento, a maioria delas (76\%) respondeu ser difícil a adesão do parceiro da gestante ao tratamento, seguido da falta de comprometimento da gestante para seguir o tratamento; $10 \%$ das enfermeiras afirmaram que não prescrevem a penicilina benzatina, dificultando o tratamento. Justificaram a ação por acreditarem ainda na necessidade de encaminhar a gestante para que o médico prescreva a medicação.

E quanto ao tratamento e abordagem de parceiros sexuais de gestantes com teste positivo para sífilis, as participantes da pesquisa citam a educação em saúde como principal estratégia, para acolher e conseguir a participação dos homens no tratamento efetivo para síflis. Das enfermeiras entrevistadas, sete (24\%) responderam que solicitam a presença do parceiro na consulta junto com a gestante. Relataram também oferecer o teste rápido para os parceiros e que realizam o tratamento na unidade; $24 \%$ das enfermeiras optam por realizar o acolhimento do casal.

\section{DISCUSSÃO}

Os dados apresentados vão ao encontro de pesquisa realizada no Ceará, onde a maioria dos enfermeiros relatou ter concluído a especialização em Saúde da Família. A capacitação e os cursos de pósgraduação propiciam ao profissional uma visão mais abrangente e segura frente às demandas de atendimento da população, interferindo na qualidade da assistência prestada e na forma de condução de questões de difícil manejo dentro da Unidade de Saúde 5 .

O estudo acima também cita a oferta do teste rápido como facilidade no manejo da síflis no prénatal, o que facilita o resultado e diagnóstico precoce. A disponibilização do teste rápido é uma estratégia oportuna para o casal frente ao início do acompanhamento prénatal, facilitando a identificação da sífilis de forma precoce, e o tratamento imediato com redução de chances de transmissão vertical da infecção $0^{5}$.

$\mathrm{Na}$ presente pesquisa observou-se facilidade quanto à disponibilidade da penicilina benzatina para o tratamento; uma resposta positiva, pois esta é a única terapia medicamentosa com eficácia comprovada e com efeito para o tratamento da sífilis em gestante em todo o mundo ${ }^{1}$.

Um estudo recente realizado em 43 países da África subsaariana comprovou a redução de óbito fetal 
e morte neonatal em gestantes portadoras de sifilis que realizaram o tratamento com penincilina benzatina. Foi realizada uma metanálise dos resultados de nascimentos em mulheres grávidas infectadas com sífilis em contextos limitados de recursos; quem??? relataram que a terapia com penicilina reduziu a incidência de óbito fetal e morte neonatal em $80 \%$ e a síflis congênita em $97 \%{ }^{6}$.

$\mathrm{O}$ tratamento recomendado para gestantes com sífilis recente (com menos de 02 anos de evolução), síflis primária, secundária e latente recente deve ser feito com penicilina $\mathrm{G}$ benzatina, na dose de 2,4 milhões UI, intramuscular em dose única. Já para síflis (com mais de 02 anos de evolução), latente tardia ou latente com duração ignorada e sífilis terciária, a dose deve ser de 7,2 milhões UI, intramuscular, dividida em três semanas consecutivas com intervalo de sete dias entre cada aplicação ${ }^{1}$

O Conselho Federal de Enfermagem estabelece que a penicilina benzatina pode ser administrada por profissionais de enfermagem no âmbito das UBS, mediante prescrição médica ou de enfermagem; e ainda, enfermeiros podem prescrever a penicilina benzatina, conforme protocolos estabelecidos pelo Ministério da Saúde, Secretarias Estaduais e Municipais de Saúde ou em rotina aprovada pela instituição de saúde"

Umapequenaparceladasenfermeiras pesquisadas cita que não prescreve e administra a penicilina na própria unidade, sendo necessária a prescrição médica. Esta realidade pode retratar o momento de adequação às novas diretrizes do Ministério da Saúde para o tratamento da sífilis em gestantes pelo município pesquisado, e ainda, pode mascarar certa insegurança por parte das profissionais em realizar o tratamento da sífilis.

O fluxograma de tratamento de sífilis em gestante indica o início do tratamento com primeira dose de penicilina benzatina imediatamente, após o diagnóstico com teste rápido, seguido de coleta de amostra para realização de teste laboratorial, e ainda, a testagem e tratamento do parceiro sexual ${ }^{1}$. O que mais uma vez reforça o tratamento a ser realizado pelo profissional que atende a gestante no momento do diagnóstico, podendo ser médico ou enfermeiro.

O rápido retorno dos resultados de exames foi citado como fator que facilita o diagnóstico e o tratamento da sífilis em gestantes. Após a chegada do exame laboratorial nas unidades e apresentando VDRL e teste treponêmico reagente, a gestante deve ser convocada juntamente com o parceiro para orientações e iniciar o tratamento. Será enviada a notificação compulsória com as datas fidedignas das tomadas de medicação. Será solicitada nova sorologia para avaliar a titulação após 21 dias do tratamento, repetindo mensalmente novas solicitações de exames para acompanhamento durante a gestação ${ }^{8}$.

Assim como nesta pesquisa, a dificuldade de tratamento e adesão por parte dos parceiros de gestantes também ficou evidenciada em outro estudo que destacou a resistência ao tratamento, tendo como desfecho a síflis congênita, pois a maioria das gestantes com síflis estava inserida num contexto socioeconômico precário, vivendo, portanto, em situações de risco e vulnerabilidade. É o caso das gestantes com parceiros presidiários ou com múltiplos parceiros, dificultando a adesão ao pré-natal e ao tratamento da doença, o que resulta na continuidade da cadeia de transmissão da síflis, comprometendo negativamente as ações e a atuação dos enfermeiros 5 .

A questão de dificuldade do tratamento do parceiro vem sendo apontada como o maior vetor desta infecção, e que o mesmo, em grande parte dos casos, não se trata junto com sua companheira, ocorrendo a reinfecção da mesma, com consequente dano em maior escala para o feto 9 .

Por isso, torna-se necessária uma atitude eficaz dos gestores e trabalhadores de saúde para captação destes parceiros, bem como a extensão da assistência do pré-natal para estes. Sendo essas as principais atitudes relatadas pelas enfermeiras desta pesquisa como estratégia para o tratamento dos mesmos. É fundamental realizar busca ativa para diagnóstico e tratamento de parceiros sexuais de gestantes com sífilis, bem como fortalecer o pré-natal do parceiro nos serviços de saúde ${ }^{1}$.

O ponto forte nas respostas das enfermeiras refere-se ao acolhimento e educação em saúde dos parceiros identificados e recrutados, corroborando com essa ideia, estudo realizado em Sobral (CE) identificou que para a boa adesão dos parceiros ao tratamento, são necessários: acolhimento, empatia e comunicação eficaz. Ações essenciais para a construção do vínculo 
entre profissionais da Atenção Básica e usuários. O vínculo é de fundamental importância para a melhoria do atendimento, pois é a partir desse componente que o enfermeiro estabelece uma relação de confiança com o usuário no cotidiano do tratamento da sífilis ${ }^{10}$.

Quanto à educação em saúde, esta é o primeiro passo para estimular a adesão ao tratamento e a responsabilização pela cura e quebra da cadeia de transmissão. A carência de informações acerca da sífilis promove a reflexão de que o conhecimento é inerente à prevenção e à adesão ao tratamento da doença e o seu desconhecimento torna a problemática da sífilis ainda maior, ocasionando sentimentos e atitudes que dificultam o processo de cura e prevenção da doença ${ }^{10}$.

A sífilis é uma doença milenar que ainda apresenta aumento de casos novos e traz riscos à saúde da gestante, do feto e do recém-nascido, portanto não se deve subestimar a doença e suas consequências para a saúde da população.

Esta pesquisa traz como limitação a visão de uma realidade local, porém a discussão reforça o que outros autores também observaram em suas realidades, ou seja, a necessidade de enfrentamento da sífilis e os desafios expostos para o controle da doença.

\section{CONCLUSÃO}

Quanto às facilidades e dificuldades para o tratamento da sífilis, constatou-se que há facilidades ligadas à ordenação das ações, à testagem e aos fluxos de atendimento recomendados pelo Ministério da Saúde. Já as dificuldades emanam da baixa adesão de parceiros sexuais em realizarem o tratamento e o acompanhamento destes pela unidade de saúde.

Em relação ao diagnóstico e ao tratamento da sífilis durante o pré-natal, conclui-se que os testes rápidos estão disponíveis no município; o laboratório atende a demanda apresentada pelas unidades de saúde. Ainda assim é necessário um avanço no tratamento realizado pela enfermeira, na totalidade dos casos, no que diz respeito à prescrição da penicilina e seu uso dentro da própria unidade básica de saúde.

Conclui-se que os desafios encontrados pelas enfermeiras em uma cidade do interior do Estado do Rio de Janeiro, para o controle da sífilis na gestação, vêm ao encontro de estudos realizados com a temática proposta e que se faz necessário o acolhimento do casal durante as consultas de pré-natal para fortalecer o controle da sífilis na atenção primária.

\section{REFERÊNCAS}

1. Ministério da Saúde (BR). Secretaria de Vigilância em Saúde. Departamento de DST, Aids e Hepatites Virais. Protocolo clínico e diretrizes terapêuticas para prevenção da transmissão vertical de hiv, sífilis e hepatites virais. Brasília: Ministério da Saúde; 2017.

2. Ministério da Saúde (BR). Secretaria de Vigilância em Saúde. Departamento de DST, Aids e Hepatites Virais. Protocolo Clínico e Diretrizes Terapêuticas para Atenção Integral às Pessoas com Infecções Sexualmente Transmissíveis. Brasília: Ministério da Saúde; 2015.

3. Ministério da Saúde (BR). Departamento de DST, Aids e hepatites viras. Boletim epidemiológico sífilis 2016. [acesso em 2017 Mar 05] Disponível em: http://www. aids.gov.br/system/tdf/pub/2016/59222/2016_030_ sifilis_ publicao 2 _ pdf_ 51905 . pdffile $=1 \&$ type $=$ node $\& i d=59222 \&$ force $=1$.

4. França IFX, Batista JDL, Coura AS, Oliveira CF, Araújo AKF, Sousa FS. Fatores associados à notificação da sífilis congênita: um indicador de qualidade da assistência pré-natal. Rev Rene. 2015;16(3):374-81.

5. Martins KMC, Silva MAM, Albuquerque IMAN , Aquino PS, Linhares MSC. O discurso dos enfermeiros sobre assistência pré-natal de gestantes com síflis. CIAIQ. 2016;2:1426-35.

6. Kuznik A, Habib AG, Manabe YC,Lamorde M. Estimating the public health burden associated with adverse pregnancy outcomes resulting from syphilis infection across 43 countries in sub-Saharan Africa. Sex Transm Dis, Bethesda, July. 2015. [acesso em 2017 Out 02]. Disponível em:https://www.ncbi.nlm. 
nih.gov/pubmed/26222749.

7. Conselho Federal de Enfermagem. Nota Técnica COFEN/CTLN No03/2017. Brasilia, 14 Jun. 2017. [acesso em 2017 Out 02]. Disponível em: http://www. cofen.gov.br/wp-content/uploads/2017/06/NOTAT\%C3\%89CNICA-COFEN-CTLN-N\%C2\%B0-03-2017. pdf.

8. Siqueira AM, Siqueira WL, Rodrigues FSM, Errante PR, Ferraz RRN. Sífilis em gestantes atendidas em unidades de saúde da região metropolitana do município de São Paulo - SP: um estudo qualitativo de casos múltiplos. Atas Cienc Saude. 2016;4(2):3146. [acesso em 2017 Set 15]. Disponível em: http:// www.revistaseletronicas.fmu.br/index.php/ACIS/ article/view/1109.

9. Santos VC, ANJOS KF. Sífilis: uma realidade prevenível. Sua erradicação, um desafio atual. Saúde e Pesqui. 2009;2(2):257-63. [acesso em 2017 Out 02]. Disponível em: http://periodicos.unicesumar. edu.br/index.php/saudpesq/article/view/1027.

10. Vasconcelos M, Guimarães R, Magalhães A, Oliveira $\mathrm{K}$, Linhares M, Albuquerque I, et al. Estratégias e Desafios dos Enfermeiros da Atenção Básica para o Tratamento Simultâneo da Síflis. CIAIQ. 2016;2: 1584-92.

Recebido em: 16/11/2017 Aceito em: 04/06/2018 